\title{
¿Producen Resultado Adverso de Género las Entrevistas Estructuradas de Selección de Personal?
}

\section{Do Structured Selection Interviews Produce Gender Adverse Impact?}

\author{
Pamela Alonso \\ Universidad de Santiago de Compostela
}

\begin{abstract}
Resumen. Estudios anteriores han demostrado la validez y la fiabilidad de las entrevistas estructuradas de selección. Sin embargo, se han obtenido resultados contradictorios sobre si este método produce impacto adverso hacia las mujeres. Este estudio es un meta-análisis que tiene como objetivo determinar si las entrevistas estructuradas producen impacto adverso según el sexo del entrevistado. Los resultados indican que no hay diferencias en las puntuaciones para hombres y mujeres en ninguna de las modalidades de la entrevista estructurada. Finalmente, se discuten las implicaciones de estos resultados, tanto para la investigación como para la práctica en selección de personal.

Palabras clave: entrevista, estructura, impacto adverso, selección de personal, género.
\end{abstract}

\begin{abstract}
Previous studies have demonstrated the validity and reliability of structured interviews for personnel selection. However, results have been contradictory on whether this method produces adverse impact against women. This study is a meta-analysis to determine if structured interviews produce adverse impact by gender of the interviewee. Findings indicate no differences in scores for men and women for any of the modalities of the structured interview. Finally, implications of these results are discussed for research and for practice in personnel selection.

Keywords: interview, structure, adverse impact, personnel selection, gender.
\end{abstract}

Diversos meta-análisis llevados a cabo en los últimos 15 años han mostrado que las entrevistas estructuradas de selección predicen tanto el desempeño laboral como el éxito en la formación y que su validez se generaliza a través de puestos, criterios y organizaciones (Huffcutt y Arthur, 1994; McDaniel et al.,1994; Salgado y Moscoso, 1995; Schmidt y Rader, 1999). Además, la entrevista es un método fiable, con valores que oscilan entre .64 para las entrevistas convencionales (Conway, Jako y Goodman, 1995) y .83 para las entrevistas conductuales estructuradas (Salgado, Moscoso y Gorriti, 2004). Este hecho adquiere mayor importancia si se tiene en cuenta que la entrevista de selección de personal es el instrumento más empleado por las empresas en sus procesos selectivos (Salgado, Viswesvaran y Ones, 2001) y que las entrevistas son el método preferido por los solicitantes en todos los países (Anderson, Salgado y Hülsheger, 2010). La entrevista adquiere así un papel fundamental en la toma de decisión de contratación, pues en la mayoría de los casos la decisión última se hace con este instrumento, siendo en algunos casos el único método de evaluación empleado (Salgado, Moscoso y Gorriti, 2004).

La investigación incluida es este artículo ha sido parcialmente financiada con cargo a la ayuda concedida al proyecto PSI2008/03617 por el Ministerio de Ciencia e Innovación. La correspondencia deberá enviarse a Pamela Alonso, Departamento de Psicología Social. E.U. Relaciones Laborales, Universidad de Santiago de Compostela, 15782 Santiago de Compostela. E-mail: pamalon@gmail.com
Aunque se suele utilizar el concepto de entrevista de selección de modo genérico, la realidad es que las entrevistas se pueden clasificar en diferentes tipos. Así, se puede hablar de dos grandes divisiones: (a) en función de su contenido y (b) en función de su formato o grado de estructura. Con respecto al primer grupo, Janz (1982) dividió las entrevistas en dos tipos de acuerdo al contenido de las preguntas: (1) convencionales y (2) conductuales. Las entrevistas convencionales se basan en preguntas sobre credenciales, logros y datos biográficos, así como descripciones sobre la experiencia y auto evaluaciones. En algunas organizaciones, la entrevista convencional dura muy pocos minutos y las preguntas no son las mismas para todos los candidatos, mientras que en otras, la duración suele ser amplia y se utiliza el mismo repertorio de preguntas para todos los entrevistados. A las primeras se les puede denominar entrevistas convencionales no estructuradas o semiestructuradas y a las últimas entrevistas convencionales estructuradas. En cuanto al segundo tipo de entrevista mencionadas por Janz, las entrevistas conductuales, éstas se centran en preguntas sobre experiencias en el trabajo y, sobre todo, descripciones de conducta. Aunque hay diversos tipos de entrevistas conductuales, todas ellas presentan algunas características en común: (1) las preguntas de la entrevista son desarrolladas a partir de un análisis previo del puesto, (2) a todos los candidatos se les hace las mismas preguntas, (3) se utilizan anclajes conductuales para evaluar a los candida- 
tos, (4) se lleva a cabo el mismo procedimiento con todos los candidatos, (5) los entrevistadores son entrenados en el uso de la entrevista y (6) la decisión de contratación no se toma hasta que han sido realizadas las entrevistas a todos los candidatos (Salgado y Moscoso 2002; Salgado y Moscoso, 2005). Los dos tipos de entrevistas conductuales más utilizadas son las entrevistas situacionales, en las que se les pregunta a los candidatos sobre cuál sería su actuación ante situaciones hipotéticas que podrían producirse en el puesto, y las entrevistas conductuales estructuradas (ECE), en las que se les pide a los candidatos que describan su comportamiento en determinadas situaciones pasadas. Otras entrevistas conductuales estructuradas, aunque menos utilizadas, serían la entrevista multimodal y la entrevista basada en el contenido del puesto. (Feild y Gatewood, 1989; Schüler y Funke, 1989).

Con respecto al formato o grado de estructura, éste puede considerarse un continuo compuesto por dos dimensiones: las cuestiones de la entrevista y la valoración de las respuestas. Con respecto a la primera dimensión, Huffcutt y Arthur (1994) hicieron una clasificación basada en cuatro niveles. El primer nivel se caracterizaría por una total ausencia de restricciones formales, el entrevistador puede plantear las preguntas que él considere oportunas durante el transcurso de cada una de las entrevistas que lleve a cabo. Es el caso de las entrevistas convencionales no estructuradas o semiestructuradas. El segundo nivel estaría formado por aquellas entrevistas en las cuales se le proporciona al entrevistador una guía de las áreas a cubrir. Un ejemplo serían las entrevistas convencionales estructuradas. El tercer nivel se caracterizaría por una especificación previa de las preguntas, aunque los entrevistadores tienen flexibilidad para adaptar la entrevista a los diferentes candidatos, se les permite a los entrevistadores elegir entre cuestiones alternativas y sondear a los solicitantes sobre cuestiones específicas. Un ejemplo de este nivel sería la entrevista de descripción de conducta de Janz (1982). El cuarto nivel implica una estandarización completa, es decir, a los solicitantes se les plantean exactamente las mismas cuestiones y en el mismo orden. Aquí se incluiría la entrevista situacional (Latham, Saari, Pursell y Campion, 1980) o la entrevista conductual estructurada (Salgado y Moscoso, 2005).

La valoración de las respuestas del candidato también es susceptible de dividirse en varios niveles progresivamente más estandarizados (Campion, Palmer y Campion, 1997; Huffcutt y Arthur, 1994). El primer nivel estaría formado por aquellas entrevistas en las cuales el entrevistador hace una evaluación global del candidato basada en la información total obtenida. Este es el tipo de valoración que se hace con las entrevistas convencionales no estructuradas o semiestructuradas o la utilizada por Ghiselli (1966). El segundo nivel lo formarían aquellas entrevistas en las cuales se realizan múltiples valoraciones referidas a cada una de las dimensiones en las que están basadas las preguntas de la entrevista o a diferentes rasgos (por ejemplo el modelo de entrevista de Janz o las entrevistas convencionales estructuradas). Y, por último, el tercer nivel se caracterizaría por la valoración independiente de cada una de las respuestas dadas por los solicitantes a las preguntas en diferentes escalas adaptadas a cada una de esas cuestiones (un ejemplo es la entrevista situacional o la entrevista conductual estructurada).

Estas diferencias entre los distintos tipos de entrevistas hacen que éstas varíen también en su frecuencia de uso y sus propiedades psicométricas (Salgado y Moscoso, 2005; Salgado, Moscoso y Gorriti, 2004). Así, la investigación meta-analítica de los últimos años ha puesto de manifiesto que las entrevistas conductuales estructuradas son uno de los mejores predictores del desempeño ocupacional y que las entrevistas convencionales, si son estructuradas, muestran unos coeficientes de validez aceptables (Saez, 2007; Salgado y Moscoso, 2005; Salgado y Moscoso, 2006; Salgado, Moscoso y Gorriti, 2004). Con respecto a su grado de uso, éste es inversamente proporcional al grado de estructuración de la entrevista (Salgado y Moscoso, 2005).

Por lo tanto, hoy en día no hay duda sobre la capacidad predictiva de cada uno de los tipos de entrevistas, pero dada su elevada tasa de uso y el papel decisorio que tienen en los procesos de selección, es necesario dilucidar otras cuestiones relevantes sobre este intrumento de selección. Una cuestión que ha sido poco investigada, y que actualmente adquiere mayor relevancia, es conocer si los diferentes tipos de entrevistas estructuradas producen resultado (impacto) adverso en función del sexo del entrevistado. Actualmente en muchos países europeos y del resto del mundo existe legislación sobre igualdad de oportunidades en el acceso al empleo y en España existen varias sentencias en los tribunales sobre casos de discriminación hacia las mujeres en los procesos de selección (Caso Solvay, Sentencia del Tribunal Superior de Justicia de Cantabria 3000/2005). A este respecto, en España se aprobó en el año 2007 la Ley Orgánica 3/2007 para la Igualdad Efectiva de Mujeres y Hombres que define en su artículo 6 los conceptos de discriminación directa e indirecta por razón de sexo. Así, se considera discriminación directa por razón de sexo "la situación en que se encuentra una persona que sea, haya sido o pudiera ser tratada, en atención a su sexo, de manera menos favorable que otra en situación comparable". En cuanto a la discriminación indirecta por razón de sexo, se define como "la situación en que una disposición, criterio o práctica aparentemente neutros pone a personas de un sexo en desventaja particular con respecto a personas del otro, salvo que dicha disposición, criterio o práctica puedan justificarse objetivamente en atención a una finalidad legítima y que los medios para alcanzar dicha finalidad sean necesarios y adecuados". Un concepto relacionado con la discriminación indirecta es el de resul- 
tado o impacto adverso, que se produce cuando la aplicación de un mismo criterio, método o procedimiento para la selección tiene un efecto negativo sobre uno o más grupos o minorías. El resultado o impacto adverso puede ser un indicio de discriminación indirecta pero, en sí mismo, es un término neutro que simplemente refleja la posibilidad de injusticia contra determinados grupos, pero no necesariamente en todas las ocasiones en las que se utilice un instrumento con impacto adverso se estaría incurriendo en discriminación (Aramburu-Zabala, 2001). En este sentido, la legislación europea y la de los EEUU exigen que los procedimientos selectivos que producen impacto adverso de género deben cumplir dos requisitos para poder utilizarse sin ser constitutivos de discriminación indirecta: ser válidos (que sean capaces de predecir el desempeño) e idóneos (que no exista otra prueba similar con igual o superior validez que no produzca impacto adverso). En estos casos se estaría hablando de resultado o impacto adverso de género no discriminatorio. Sin embargo, si no se cumplen estos requisitos, el uso de una prueba con impacto adverso podría considerarse discriminatorio. En el método que nos ocupa, de demostrarse la existencia de impacto adverso en contra de las mujeres en el uso de las entrevistas de selección, se podría propiciar la aparición de demandas por discriminación indirecta, al entenderse que vulneraría los principios de igualdad entre mujeres y hombres establecidos por la Constitución Española y recientemente regulados por la Ley 3/2007.

El impacto adverso ha sido ampliamente estudiado en relación con algunos instrumentos de selección. Por ejemplo, el efecto de los tests de habilidad mental general sobre los negros e hispanos. La investigación ha mostrado que los blancos puntúan 1 desviación típica por encima de los negros y 0.7 desviaciones típicas por encima de los hispanos (Hunter y Hunter, 1984; Schmitt et al., 1996). Con respecto al género, los resultados de diversos estudios han mostrado que no existe impacto adverso cuando se trata de habilidad mental general y se produce un pequeño impacto negativo hacia las mujeres en las pruebas de habilidad numérica o espacial y hacia los hombres en el caso de la habilidad verbal (Hough, Oswald y Ployhart, 2001). Con respecto a las entrevistas, la investigación ha sido mucho más escasa y básicamente se ha centrado en los efectos sobre la raza. Así, por ejemplo, Huffcutt y Roth (1998) en una investigación meta-analítica encontraron que la entrevista no tenía un gran impacto negativo sobre las minoría étnicas $(\mathrm{d}=.25)$ y que las entrevistas conductuales estructuradas tenían un impacto menor que las entrevistas poco estructuradas. PrewettLivingston y Feild (1999) encontraron que el uso de entrevistas conductuales estructuradas reducía el potencial efecto que pueden tener los sesgos raciales. Con respecto al impacto adverso en función del género, Arvey (1979) encontró que las mujeres recibían puntuaciones inferiores a las de los hombres en las entrevistas, si bien su investigación sólo se tenían en cuenta entrevistas no estructuradas. Por su parte, Huffcutt, Conway, Roth y Stone (2001) encontraron que apenas había diferencias en las valoraciones globales de la entrevista entre hombres y mujeres $(\mathrm{d}=0.6)$, pero sí cuando lo que se evaluaba eran constructos específico tales como, por ejemplo, la inteligencia general o las habilidades sociales. En el primer caso, el efecto negativo se produce en las mujeres y en el segundo en los hombres. Analizando por separado las entrevistas en función de su grado de estructura, los resultados mostraron que las entrevistas con alta estructura no producían impacto adverso. Sin embargo, en las entrevistas de baja estructura encontraron una $\mathrm{d}=.23$, lo que sugiere que este tipo de entrevistas sí producen cierto impacto adverso hacia las mujeres. Por su parte, Moscoso (2000), en su revisión sobre la entrevista de selección, concluyó que las entrevistas producen menor impacto adverso que otros instrumentos de selección y este pequeño efecto podía reducirse con el uso de entrevistas conductuales estructuradas, si bien señaló que la investigación al respecto aún era muy escasa, tal y como han indicado previa y posteriormente otros autores.

No obstante, han pasado diez años desde estas dos revisiones de la literatura (Huffcutt, Conway, Roth y Stone, 2001; Moscoso, 2000) y desde entonces se han publicado más estudios primarios, pero no se ha llevado a cabo una nueva integración cuantitativa de los resultados. En consecuencia, el objetivo de este estudio es comprobar si las entrevistas de selección estructuradas, en cualquiera de sus modalidades, producen resultado o impacto adverso en función del género. Sobre la base de la literatura previa planteamos la hipótesis siguiente: Las entrevistas estructuradas de selección de personal no producen impacto adverso independientemente del tipo de entrevista del que se trate.

\section{Método}

\section{Búsqueda de estudios}

El objetivo de la búsqueda fue encontrar el mayor número posible de investigaciones con datos sobre los efectos del sexo del entrevistado sobre la puntuación final en la entrevista. Por tanto, para incluir los estudios en el meta-análisis, se siguieron dos criterios: (1) que el estudio publicase el tamaño del efecto del sexo sobre la puntuación final en la entrevista u otro tipo de datos que permitiesen calcularlo (por ejemplo, el tamaño, la media y la desviación típica de los dos grupos, la correlación entre el sexo y la puntuación final, etc.), (2) que el objetivo de la entrevista fuese examinar el ajuste del candidato con el puesto.

Para llevar a cabo la búsqueda, se emplearon cuatro estrategias diferentes: (a) búsqueda en las bases de datos electrónicas: PsycInfo, Google Scholar y SAGE, 
(b) búsqueda artículo por artículo en Journal of Applied Psychology, Personnel Psychology y en International Journal of Selection and Assessment, (c) se comprobaron las referencias de los artículos encontrados en las búsquedas anteriores para localizar nuevos estudios y (d) se contactó con algunos investigadores en este ámbito con la finalidad de conseguir datos todavía no publicados.

Finalmente, se encontraron 11 investigaciones (12 muestras independientes) que podían ser incluidas en el meta-análisis, de los cuales ocho son artículos publicados y tres son tesis doctorales.

\section{Procedimiento}

Una vez seleccionados los estudios que formarían parte del meta-análisis, los resultados de los mismos fueron codificados en una base de datos recogiendo la siguiente información: (a) referencia del artículo, (b) tipo de entrevista, (c) fiabilidad de la entrevista, (d) características de la muestra (tamaño inicial, tamaño final, total hombres, total mujeres, etc.) y (e) las medidas que permitían calcular el tamaño del efecto (d de Cohen; media y desviación típica de cada grupo; correlación entre entrevista y sexo; etc.).

Las diferentes muestras fueron clasificadas, en función del contenido de la entrevista, en las siguientes categorías: (1) convencional o (2) conductual, y dentro de estas últimas: (2a) situacional o (2b) conductual estructurada (ECE). Este proceso fue llevado a cabo por dos investigadores de modo independiente, obteniendo un grado de acuerdo en sus clasificaciones del $100 \%$ (Kappa de Cohen=1). Varios de los estudios incluidos en el meta-análisis utilizaron diferentes tipos de entrevistas con la misma muestra de sujetos. Por este motivo, en el meta-análisis global de la entrevista, para evitar que las muestras se duplicasen, se procedió a promediar los resultados en aquellos estudios que utilizaron más de una entrevista, incluyéndolos en el meta-análisis como una única muestra. Sin embargo, cuando se realizó el meta-análisis en función del tipo de entrevista, se tuvieron en cuenta los resultados encontrados para cada tipo concreto. Por este motivo, la suma de los estudios incluidos en los meta-análisis en función del tipo de entrevista, es superior, conjuntamente, al número de estudios incluido en el meta-análisis del total de las entrevistas. Así, para el meta-análisis de las entrevistas convencionales se obtuvieron 4 muestras y para el meta-análisis de las conductuales 9 . Dentro de estas últimas, fueron analizados por separado los datos correspondientes a las entrevistas conductuales estructuradas (7 muestras) y a las entrevistas situacionales (5 muestras).

Una vez recogidos los datos, se calculó el tamaño del efecto medio ponderado por el tamaño de la muestra para los diferentes tipos de entrevista. A continuación, se realizó el meta-análisis utilizando el método desarrollado por Hunter y Schmidt (2004). En este meta-análisis se corrigió el tamaño medio del efecto observado por falta de fiabilidad y por restricción directa en el rango.

\section{Estimación del tamaño del efecto}

En la mayor parte de los estudios incluidos en el meta-análisis se había calculado la media y desviación típica de los dos grupos, por lo que en ese caso se procedió a calcular la d de Cohen (Cohen y Cohen, 1983). En el resto de los estudios, los datos se expresaron en forma de correlación entre el sexo del candidato y la puntuación final en la entrevista, por lo que se procedió a su transformación a d de Cohen a través de la fórmula desarrollada por Hunter y Schmidt (2004).

\section{Fiabilidad del criterio}

La fiabilidad del criterio fue estimada (a) teniendo en cuenta los coeficientes publicados en los estudios incluidos en el meta-análisis y (b) utilizando los coeficientes publicados en meta-análisis anteriores sobre la fiabilidad de las entrevistas de selección (Conway, Jako y Goodman, 1995; Salgado, Moscoso y Gorriti, 2004). Se utilizaron los coeficientes de fiabilidad interjueces correspondientes a cada tipo de entrevista. En la Tabla 1, se pueden ver los coeficientes de fiabilidad promedio para cada tipo de entrevista.

Tabla 1. Fiabilidad en función del tipo de entrevista.

\begin{tabular}{lllr}
\hline Tipo entrevista & $\mathrm{r}_{\mathrm{xx}}$ & $\mathrm{SD}_{\mathrm{r}}$ & $\mathrm{K}$ \\
\hline Total & .82 & .06 & 12 \\
Convencional & .75 & .04 & 4 \\
Conductual & .85 & .04 & 9 \\
$\quad$ ECE & .86 & .04 & 7 \\
$\quad$ Situacional & .82 & .01 & 5 \\
\hline
\end{tabular}

Nota. $\mathrm{r}_{\mathrm{rx}}=\mathrm{valor}$ medio de la fiabilidad de la entrevista; $\mathrm{SD}=$ =desviación estándar de la fiabilidad; K=número de coeficientes de fiabilidad.

\section{Restricción en el rango}

En varias de las muestras se había producido restricción directa en el rango, dado que la muestra final era más pequeña que la muestra inicial. Por tanto, se procedió a la corrección por restricción en el rango. Para ello, se halló el valor promedio de esta restricción en función del tipo de entrevista, calculando el valor de la restricción en cada uno de los estudios, utilizando la razón de selección de cada una de las muestras. En la Tabla 2 se pueden observar los valores promedio de la restricción en el rango para cada tipo de entrevista. 
Tabla 2. Restricción en el rango en función del tipo de entrevista

\begin{tabular}{llll}
\hline Tipo entrevista & $\mathrm{u}$ & $\mathrm{SD}_{\mathrm{r}}$ & $\mathrm{K}$ \\
\hline Total & .89 & .15 & 12 \\
Convencional & .89 & .13 & 4 \\
Conductual & .88 & .16 & 9 \\
$\quad$ ECE & .85 & .17 & 7 \\
$\quad$ Situacional & .91 & .09 & 5
\end{tabular}

Nota. $\mathrm{u}=$ valor medio del ratio de restricción en el rango; $\mathrm{SD}_{\mathrm{u}}=$ desviación estándar del ratio de restricción en el rango; $\mathrm{K}=$ número de coeficientes.

Para poder llevar a cabo la corrección por restricción en el rango en el tamaño del efecto, todas las puntuaciones d de Cohen fueron transformadas a correlaciones a través de la fórmula propuesta por Hunter y Schmidt (2004): $r=d / 2$. Por este motivo, el procedimiento meta-analítico llevado a cabo ha sido el correspondiente al de un meta-análisis de correlaciones. Una vez realizado el análisis, los resultados obtenidos se transformaron de nuevo a d de Cohen empleando la fórmula anterior.

\section{Resultados}

La Tabla 3 muestra los resultados para cada tipo de entrevista. En primer lugar, podemos observar (de izquierda a derecha), el número de estudios y el tamaño de las muestras. En el primer meta-análisis realizado, sobre el total de las entrevistas, el número de estudios es de 12, con una muestra total de 32,525 sujetos. Para el meta-análisis de las entrevistas convencionales se contó con 4 muestras, con un total de 19,457 individuos y para las conductuales, con 9 estudios y 31,434 participantes. A continuación, en las cinco columnas siguientes, se pueden ver las medias de las correlaciones observadas ponderadas por el tamaño de la muestra, las desviaciones típicas observadas, las varianzas observadas, la varianza debida a errores artifactuales y el porcentaje de la varianza observada debida a error. En las siguientes tres columnas se puede ver la correlación verdadera (corregida por restricción en el rango y falta de fiabilidad), la varianza de la correlación ver- dadera y el valor mínimo de credibilidad del $90 \%$ de la correlación verdadera.

Todas las correlaciones resultantes en los diferentes meta-análisis son de signo negativo, lo que supone puntuaciones más bajas para el grupo de los hombres que para el grupo de las mujeres. Los resultados muestran correlaciones bajas en todos los casos. Así, en el meta-análisis del total de las entrevistas, es de -.07, con un $9.59 \%$ de la variabilidad observada debida a los errores artifactuales y un valor mínimo de credibilidad en un intervalo del $90 \%$ de .030 , con lo que se incluiría el 0 en el intervalo de credibilidad. A la vista de los resultados, debemos concluir que no existen diferencias entre los dos grupos; es decir, no se produce ningún efecto en las puntuaciones en función del sexo. Según el tipo de entrevista, encontramos que la correlación más alta corresponde a las entrevistas convencionales con un valor de -. 15 , lo que supone un $2.25 \%$ de la varianza explicada. La variabilidad observada debida a errores artifactuales fue del $10.65 \%$ y el valor mínimo de credibilidad del $90 \%$ fue de -.049 , lo que indica que en este caso los resultados sean claramente generalizables. En el conjunto de las entrevistas conductuales, la correlación operativa resultante es de -.07 , con un porcentaje de la varianza debido a errores artifactuales del $13.62 \%$ y un valor de credibilidad del $90 \%$ de .004 . Este resultado debe ser interpretado del mismo modo que en el caso del conjunto de las entrevistas. Es decir, una vez más, se observa que no hay diferencias en las puntuaciones en función del sexo.

Analizando por separado los dos tipos de entrevistas conductuales, se puede observar que en las ECE, el valor de la correlación operativa es de -.09 , con un 9.92\% de la varianza observada debida a errores artifactuales y un valor credibilidad del $90 \%$ de .00 . En cuanto a las entrevistas situacionales, el valor de la correlación operativa es de.- .09 , valor que coincide con el valor mínimo de credibilidad del $90 \%$, dado que el porcentaje de la varianza observada debida a errores artifactuales es del 100\%, lo que evidencia la generalización de los resultados encontrados para este tipo de entrevistas. En la Tabla 4 podemos observar los resultados finales del meta-análisis expresados en tamaño del efecto (d de Cohen).

Tabla 3. Resultados del meta-análisis de la correlación entre el sexo del entrevistado y la puntuación en la entrevista.

\begin{tabular}{lrrrrrrrrr}
\hline & $K$ & $N$ & $r$ & $S D_{r}$ & $S_{A R T}^{2}$ & $\% V E$ & $\rho$ & $S_{p}^{2}$ & $90 \% V C$ \\
\hline Total & 12 & 32525 & -.06 & .070 & .0005 & 9.59 & -.07 & .007 & .030 \\
$\quad$ Convencional & 4 & 19457 & -.11 & .063 & .0004 & 10.65 & -.15 & .059 & -.049 \\
$\quad$ Conductual & 9 & 31434 & -.06 & .053 & .0004 & 13.62 & -.07 & .049 & .004 \\
$\quad$ ECE & 7 & 30895 & -.07 & .064 & .0004 & 9.92 & -.09 & .061 & .000 \\
$\quad$ Situacional & 5 & 18935 & -.07 & .015 & .0003 & 100.00 & -.09 & .000 & -.090 \\
\hline
\end{tabular}

Nota. El signo negativo en las correlaciones muestra una menor puntuación en el grupo de los hombres. K=número de estudios; N=tamaño total de la muestra; r=correlación observada ponderada por el tamaño de la muestra; $\mathrm{SD}_{\mathrm{r}}=$ desviación estándar observada; $\mathrm{S}_{\mathrm{ART}}^{2}=$ varianza debida a errores artifactuales; \%VE=porcentaje de la varianza observada debida a errores artifactuales; $\rho=$ correlación verdadera; $\mathrm{S}_{p}^{2}=$ varianza de la correlación verdadera; $90 \% \mathrm{VC}=$ valor de credibilidad. 
Tabla 4. Resultados del meta-análisis del tamaño del efecto del sexo sobre las puntuaciones en las entrevistas estructuradas

\begin{tabular}{lrrrc}
\hline & $K$ & $N$ & $d_{c}$ & $90 \% V C$ \\
\hline Total & 12 & 32525 & -.14 & .06 \\
$\quad$ Convencional & 4 & 19457 & -.30 & -.10 \\
Conductual & 9 & 31434 & -.14 & .01 \\
$\quad$ ECE & 7 & 30895 & -.18 & .00 \\
$\quad$ Situacional & 5 & 18935 & -.18 & -.18 \\
\hline
\end{tabular}

Nota. $\mathrm{K}=$ número de estudios; $\mathrm{N}=$ muestra total; $\mathrm{d}$ =tamaño del efecto corregido medio ponderado. $90 \% \mathrm{CV}=$ valor de credibilidad.

Para apoyar la generalización de los resultados obtenidos, debemos tener en cuenta el tamaño de la muestra. Desde la teoría del muestreo, las muestras de más de 400 personas se consideran muestras grandes (Hunter y Schmidt, 2004), ya que garantizan cierta estabilidad en las estimaciones de muestreo. Como puede observarse, todos los meta-análisis realizados incluyen muestras que oscilan entre 18,935 sujetos y 32,525. En resumen, los resultados de este meta-análisis demuestran que no se produce impacto adverso en las entrevistas de selección de personal estructuradas, independientemente del tipo de entrevista, lo confirma nuestra hipótesis de partida.

\section{Discusión}

La posibilidad de que las entrevistas de selección produzcan resultados o impacto adverso hacia grupos protegidos por ley, normalmente grupos minoritarios, es una cuestión de interés tanto desde el punto de vista teórico, como desde el punto de vista aplicado y legal. Desde el punto de vista teórico es relevante saber si un determinado procedimiento de medida, la entrevista de selección en esta investigación, produce distintos resultados para grupos diferentes y, en consecuencia, se requiere una explicación sustantiva de dicho fenómeno que permita avanzar en el conocimiento del patrón de relaciones entre las variables medidas, es decir, predictores y criterios. Por otra parte, dado que en muchos países, y entre ellos España, existen normas legales que protegen a determinados grupos de potenciales resultados adversos derivados de la utilización de un método aparentemente neutro hacia ese grupo, desde un punto de vista práctico y aplicado es fundamental conocer si dicho método produce tales resultados adversos y, en caso de que pueda producirlos, en que cuantía los produce.

Esta cuestión había sido examinada empíricamente hace ya más de tres décadas por Arvey (1979), quien había encontrado que las entrevistas de selección producían resultados adversos para las mujeres. Sin embargo, un examen detallado de los estudios analizados por este autor indica que en su mayoría las entrevistas a las que este investigador hacía referencia eran entrevistas no estructuradas y tan sólo un número pequeño eran entrevistas estructuradas, aunque de la modalidad hoy conocida o denominada convencional. Además, entre los estudios incluidos por Arvey en su revisión, un número importante de ellos se realizaron en el contexto de laboratorio, con sujetos simulados ("entrevistados de papel"), y con entrevistadores no profesionales, sino que en distintos casos eran estudiantes.

Desde el estudio pionero de Arvey, se han producido un número importante de avances en selección de personal en general y particularmente en lo referente a la entrevista de selección. Con posterioridad a la fecha del trabajo de Arvey, se han propuesto diversas modalidades de entrevistas estructuradas que tienen la característica común de basarse en la conducta del entrevistado para tomar una decisión. Entre tales entrevistas, como se ha señalado en páginas precedentes, se encuentran las entrevistas situacionales, las entrevistas de descripción de conducta, las entrevistas multimodales, las entrevistas basadas en el contenido de puestos y las entrevistas conductuales estructuradas. Ninguna de estas modalidades de entrevista fue considerada en el estudio de Arvey, por lo que las conclusiones del mismo, es decir, que las entrevistas producen resultados adversos para las mujeres, no puede ser aplicado sin más a estos nuevos tipos de entrevistas.

Además, los nuevos desarrollos metodológicos en la entrevista se han visto acompañados por estudios sobre su fiabilidad y validez predictiva. Tales estudios han mostrado que las nuevas técnicas de entrevista mostraban una alta fiabilidad y una notable validez, lo que contrastaba claramente con las conclusiones de todas las revisiones previas sobre la fiabilidad y la validez de la entrevista de selección que habían llegado a la conclusión de que esta herramienta era poco fiable y de escasa o nula validez (Conway, Jako y Goodman, 1995; Hunter y Hunter, 1984; Mayfield, 1964; Salgado, 1999; Salgado, Moscoso y Gorriti, 2004; Salgado, Viswesvaran y Ones, 2001; Schmidt y Hunter, 1998; Schmitt, 1976; Ulrich y Trumbo, 1982; Wagner, 1949; Wrigth, 1969). Por otra parte, para determinar con precisión si las entrevistas, independientemente de su modalidad, producen resultados adversos se requiere que los estudios se realicen en contextos naturales, en otras palabras, que los entrevistados sean auténticos solicitantes de empleo y que los entrevistadores sean auténticos entrevistadores que van a tomar un decisión de contratación. A lo anterior hay que añadir que en España la aparición de la Ley Orgánica 3/2007, de 22 de marzo, para La Igualdad Efectiva de Mujeres y Hombres, impone cautelas y restricción al uso indiscriminado de procedimientos de selección y que aquellas organizaciones que utilicen instrumentos que produzcan impacto adverso podrían ser demandadas por aquellos candidatos que han sido excluidos del proceso selectivo, alegando que su proceso de selección produce discriminación indirecta. 
Por tanto, una investigación que examinase el posible impacto adverso de las nuevas modalidades de entrevista, que genéricamente reciben la denominación de entrevistas estructuradas parecía totalmente indicada.

La revisión de la literatura científica condujo a la evidencia que las entrevistas estructuradas son más objetivas, dado que a todos los candidatos se les evalúa a partir de las mismas preguntas y, por tanto, se utilizan los mismos criterios. En consecuencia se planteó la hipótesis de la entrevista estructurada produce el mismo efecto para hombres y mujeres y, en definitiva, no muestra resultados o impacto adverso.

Los resultados de los diversos meta-análisis incluidos en este estudio han apoyado empíricamente la hipótesis avanzada según la cual las entrevistas estructuradas, sean convencionales o conductuales, no producen impacto adverso, pues no se encuentran diferencias en las puntuaciones de los candidatos en función de su sexo. Por tanto, el uso de este instrumento de selección no produce discriminación indirecta $\mathrm{y}$, en consecuencia, no infringe la Ley de Igualdad Efectiva de Mujeres y Hombres.

\section{Implicaciones para la práctica}

Los resultados encontrados en este meta-análisis son especialmente importantes habida cuenta que se trata del instrumento más empleado por las organizaciones para la selección de sus trabajadores. Las organizaciones deben evitar que sus procesos selectivos produzcan discriminación, y por lo tanto, emplear aquellas herramientas que garanticen la igualdad de oportunidades. Ante los resultados encontrados, nuestra recomendación a los profesionales de la selección de personal es que utilicen entrevistas estructuradas, en especial entrevistas conductuales estructuradas, y eviten el uso de las entrevistas no estructuradas. De este modo, no sólo se verá incrementada la fiabilidad y validez de la entrevista (Salgado, Viswesvaran y Ones, 2001; Schmidt y Hunter, 1998), sino que además, se asegurarán de que la entrevista que están empleando no produce impacto adverso y, por tanto, estarían respaldados ante una posible demanda por discriminación indirecta por razón de sexo.

\section{Limitaciones del estudio}

A pesar de que la muestra total de este estudio es muy amplia, el número de estudios es limitado. No debemos olvidar que la mayor parte de la muestra proviene únicamente de dos estudios, por lo que el resultado final se ha visto condicionado por los resultados encontrados en estos. Debemos recordar que estos estudios han sido realizados en un país concreto, con una cultura específica, por lo que una ampliación del número de estudios y del tamaño de la muestra podría conducir a una mayor variabilidad en los resultados. Por tanto, se recomienda la realización de más estudios sobre este tema en diferentes países, en diferentes tipos de organizaciones y para puestos de diferentes categorías, para de este modo, poder comprobar que los resultados se mantienen estables.

En resumen, el meta-análisis llevado a cabo ha mostrado que las entrevistas estructuradas de selección de personal, ya adopten una modalidad convencional o una modalidad conductual, no producen resultados adversos para las mujeres por lo que se recomienda su uso en los procesos de contratación.

\section{Extended Summary}

Several meta-analyses conducted in the past fifteen years showed that selection interviews predict job performance and training success, and that its validity is generalized across jobs, criteria, and organizations (Huffcutt \& Arthur, 1994; McDaniel et al., 1994; Salgado \& Moscoso, 1995; Schmidt \& Rader, 1999). Furthermore, interview is a reliable method, with values ranging from .64 for conventional interviews (Conway, Jako \& Goodman, 1995) to .83 for structured behavioral interviews (Salgado, Moscoso \& Gorriti, 2004). On the other hand, recent meta-analytic research has shown that a specific type of interviews, the structured behavioral interviews, are one of the best predictors of occupational performance, and that conventional interviews, if they are structured, show acceptable validity coefficients (Saez, 2007; Salgado \& Moscoso, 2005; Salgado \& Moscoso, 2006; Salgado, Moscoso \& Gorriti, 2004). With respect to their degree of use, it is inversely proportional to the degree of structuring of the interview (Salgado \& Moscoso, 2005).
An issue which has not been researched enough is whether the various types of structured interviews produce gender adverse impact. Currently there exist legislation on equal opportunities in access to employment in many countries. In this respect, Spain approved the Organic Law 3/2007 for Effective Equality of Women and Men, which defined the concepts of direct and indirect discrimination based on gender. A related concept to indirect discrimination is adverse impact, which occurs when the method or procedure used for selection has a negative effect on one or more groups or minorities. Discrimination is not necessarily produced when using an instrument with adverse impact because discrimination is a legal concept and adverse impact is a statistical issue (Aramburu-Zabala, 2001). In this respect, legislation requires that selection procedures must meet two requirements to be used without constituting indirect discrimination: be valid (they are able to predict the performance) and suitable (there is no other similar 
procedure with equal or greater validity that does not cause adverse impact).

With respect to interviews, research on the adverse impact has mainly been focused on the effects of race (Huffcutt \& Roth, 1998; Prewett-Livingston \& Field, 1999). Regarding to gender, Arvey (1979) found that women received smaller scores than those of men in the interviews, although his review only considered unstructured interviews. For its part, Huffcutt, Conway, Roth, and Stone (2001) found that there was small differences in overall ratings of the interview between men and women $(\mathrm{d}=0.6)$, when specific constructs, such as, general intelligence or social skills, were assessed. In the first case, the negative effect occurs in women and the second in men. When analyzing the interviews according to their degree of structure, the results showed that highly structured interviews did not produce adverse impact. However, interview with low structure produced adverse impact against women $(\mathrm{d}=.23)$. For its part, Moscoso (2000), in his review of the selection interview, concluded that interviews produced less gender adverse impact than other instruments of selection and that this small effect could be reduced with the use of structured behavioral interviews.

However, at present, no study has systematically examined the potential gender adverse impact of all structured interview types. Consequently, the aim of this study is to meta-analytically determine whether the structured selection interviews, in all its forms, produce gender adverse impact. Based on previous literature, the following hypothesis is advanced: Selection structured interviews do not produce gender adverse impact regardless of the type of interview.

\section{Method}

\section{Study Search}

In order to include studies in the database, they should fulfill two criteria: (1) that the study included effect sizes of gender on the total score of the interview or other data used to calculate that effect size, (2) that the purpose of the interview was to examine candidate fit with the job. Finally, eleven studies were found (12 independent samples) that could be included in the meta-analysis.

\section{Procedure}

The studies were classified, based on the content of the interview, in the following categories: (1) conventional, and (2) behavioral, and this last one into (2a) situational, (2b) behavioral structure interview. This process was independently carried out by two researchers, obtaining an agreement of $100 \%$ in their classification. Several of the studies included in the meta-analysis used more than one type of interview on the same sample of subjects. In order to avoid duplicating the sample size, the results were averaged in these studies and this figure was used in the meta-analysis. For this reason, the sum of the studies included in specific meta-analysis for each interview type is larger than the number of studies in the meta-analysis for structured interview as a whole. Thus, in the case of conventional interviews were obtained four samples and for the behavioral interviews we obtained nine. Moreover, within the latter, were analyzed separately the data for structured behavioral interviews (7 samples) and situational interviews (5 samples).

Once data were collected, the mean effect size weighted by sample size was calculated for the different types of interview. Next, we performed the metaanalysis using the method of Hunter and Schmidt (2004). The observed effect size was corrected for by criterion unreliability and direct range restriction.

\section{Effect size estimate}

Most studies calculated the mean and standard deviation of the two groups, so in these cases Cohen's d (Cohen \& Cohen, 1983) was calculated. In the other cases, data were expressed as a correlation, so, in this case, the value was transform to $d$ through the formula given by Hunter and Schmidt (2004).

\section{Reliability criterion}

The reliability of the criterion was estimated from the coefficients published in the studies included in this meta-analysis and from the coefficients published in previous meta-analysis on the reliability of selection interviews (Conway, Jako \& Goodman, 1995; Salgado, Moscoso \& Gorriti, 2004). The reliability coefficients which have been taken into account are interrater ones. Table 1 shows the average reliability coefficient for each type of interview.

\section{Restriction in the range}

Several studies showed direct restriction on the range, so we proceeded to correct it. Table 2 shows the average values of the restriction on the range for each type of interview. To carry out this correction, all of Cohen's d scores were transformed to correlations using the formula proposed by Hunter and Schmidt (2004). For this reason, the meta-analytical procedure carried out was that corresponding to a meta-analysis of correlations.

\section{Results}

Table 3 shows the results for each type of interview. First, it can be seen (from left to right), the number of 
studies and sample sizes. In the first meta-analysis on the total of the interviews, the number of studies is 12 , with cumulated sample of 32,525 subjects. There are 4 samples for the meta-analysis of conventional interviews with a total of 19,457 individuals. In the case of behavioral interviews, the number of independent studies was 9 and with 31,434 participants. In the next five columns, it can be seen the average of observed correlations weighted by sample size, the observed standard deviations, the observed variance, the variance due to artifactual errors, and the percentage of the observed variance due to artifactual error. In the last three columns, it appears the true correlation (corrected for range restriction and unreliability), the variance of the true correlation and the $90 \%$ credibility value.

All correlations are negative, implying smaller scores for men than for women. Moreover, the results show low correlations in all cases. Thus, the effect size is -.07 for the global meta-analysis, with a $9.59 \%$ of the observed variability due to artifactual errors and the $90 \% \mathrm{CV}$ of .030 . In view of the results, we conclude that there are not differences between the two groups; in other words, that there are no effect of gender on scores. Depending on the type of interview, we find that the correlation is larger in conventional interviews with a value of -.15 . The observed variability due to artifactual errors was $10.65 \%$ and the $90 \% \mathrm{CV}$ was -.049. Taken together all behavioral interviews, the operational correlation was -.07 , with a percentage of the variance due to artifactual errors of $13.62 \%$ and the $90 \% \mathrm{CV}$ of .004 . That is, once again, there is no difference in scores by gender.

Analyzing separately the two types of behavioral interviews, it can be seen that for the structured behavioral interview, the correlation value was -.09 , with a $9.92 \%$ of the variance observed due to artifactual errors and the $90 \% \mathrm{CV}$ of .00. For situational interviews, the operational correlation was -.09 and the percentage of the observed variance due to artifactual errors was $100 \%$. Table 4 shows the final results of the meta-analysis expressed as Cohen's d.

As a support for the generalization of the results, we must take into account the size of the sample. From sampling theory, samples of more than 400 people are considered large samples (Hunter \& Schmidt, 2004), as it ensures some stability in the estimates of sampling. As shown, all the meta-analyses were performed with samples of between 18,935 and 32,525 subject. In summary, the results of the meta-analyses show that there is no gender adverse impact on structured selection interviews, regardless of the kind of interview, confirms our hypothesis.

\section{Discussion}

The question whether selection interviews produce adverse impact against protected groups is an issue of interest both from theoretical and legal point of views. From the theoretical point of view is relevant to know whether the selection interview produces different results for different groups. In addition, from a practical point of view, there are laws and regulations that protect certain groups against potential adverse impact resulting from the use of a selection method apparently neutral. Therefore, from a practical and applied point of view, is essential knowing whether this method produces adverse impact and in what amount.

The results of various meta-analyses included in this study supported empirically the hypothesis advanced, i.e. that the structured interviews (conventional or behavioral) do not cause gender adverse impact, as there are no differences in the scores of the candidates depending on the sex. Therefore, the use of this tool does not produce indirect discrimination and does not violate the Act Effective Equality of Women and Men.

\section{Implications for practice}

The findings of this meta-analyses are particularly important given that interview is the most used instrument by organizations selecting employees. Organizations must ensure that their selection processes do not result in discrimination, and, therefore, they must use tools that ensure equal opportunities. Given the results, our recommendations are to use structured interviews in order to hiring personnel, especially structured behavioral interviews, and prevent the use of unstructured interviews. Thus, not only will increase the reliability and validity of the interview (Salgado, Viswesvaran \& Ones, 2001, Schmidt \& Hunter, 1998), but also will ensure that the interview does not produce gender adverse impacts.

\section{Study Limitations}

Although the total sample of this study is very large, the number of studies is limited. Two studies contributed with two very large samples and it must be remembered that these studies have been conducted in a particular country, with a specific culture, so an increase in the number of studies and sample size could lead to greater variability in the results. Therefore, we recommend performing additional studies on this subject in different countries, different types of organizations and different jobs, in order to verity that the results are stable.

In summary, the meta-analysis conducted has shown that structured interviews of personnel selection, conventional or behavioral, do not produce adverse outcomes for women, so their use for selection processes is recommended. 


\section{Referencias}

Las referencias marcadas con un asterisco indican los estudios que han sido incluidos en el meta-análisis.

Anderson, N., Salgado, J. F. y Hülsheger, U. R. (2010). Applicant reactions in selection: Comprehensive metaanalysis into reaction generalization versus situational specificity. International Journal of Selection and Assessment, 18, 291-304.

Aramburu-Zabala Higuera, L. (2001). Adverse impact in personnel selection: The legal framework and test bias. European Psychologist, 6, 103-111.

Arvey, R. D. (1979). Unfair discrimination in the employment interview: legal and psychological aspects. Psychological Bulletin, 86, 736-765.

*Arvey, R. D., Miller, H. E., Gould, R. y Burch, P. (1987). Interview validity for selecting sales clerks. Personnel Psychology, 40, 1-12.

*Berges, A. (2010). Validación de procedimientos de selección de personal en la policía local. Tesis doctoral no publicada. Universidad de Zaragoza.

Campion, M. A., Palmer, D. K. y Campion, J. E. (1997). A review of structure in the selection interview. Personnel Psychology, 50, 655-702.

Cohen, J. y Cohen, P. (1983). Applied multiple regression/ correlation analysis for the behavioral sciences. Hillsdale, NJ: Erlbaum.

Conway, J. M., Jako, R. A. y Goodman, D. F. (1995). A meta-analysis of interrater and internal consistency reliability of selection interviews. Journal of Applied Psychology 80, 565-579.

Feild, H. S. y Gatewood, R. D. (1989). Development of a selection interview: A job context method. En. R. W. Eder y G. R. Ferris (eds.). The employment interview: Theory, research and practice. Beverly Hill, CA: Sage.

Ghiselli, E. E. (1966). The validity of a personnel interview. Personnel Psychology, 19, 389-394.

*Goldberg, C. y Cohen, D. J. (2004). Walking the walk and talking the talk: Gender differences in the impact of interviewing skills on applicant assessment. Group \& Organization Management, 29, 369-384.

Hough, L. M., Oswald, F. L. y Ployhart, R. E. (2001). Determinants, detection and amelioration of adverse impact in personnel selection procedures: Issues, evidence and lessons learned. International Journal of Selection and Assessment, 9, 152-194.

Huffcutt, A. I. y Arthur, W. (1994). Hunter and Hunter (1984) revisited: Interview validity for entry-level jobs. Journal of Applied Psychology, 79, 184-190.

Huffcutt, A.I., Conway, J.M., Roth, P.L. y Stone, N.J. (2001). Identification and meta-analytic assessment of psychological constructs measured in employment interviews. Journal of Applied Psychology, 86, 897-913.

Huffcutt, A. I. y Roth, P. L. (1998). Racial group differences in employment interview evaluations. Journal of Applied Psychology, 83, 179-189

Hunter, J. E. y Hunter, R. F. (1984). Validity and utility of alternative predictors of job performance. Psychological Bulletin, 96, 72-98.
Hunter, J. E. y Schmidt, F. L. (2004). Methods of metaanalysis: Correcting Error and Bias in Research Findings. Newbury Park, CA: Sage.

Janz, T. (1982). Initial comparisons of patterned behavior description interviews versus unstructured interviews. Journal of Applied Psychology, 67, 577-580.

*Kluemper, D. H. (2006). An examination of ability-based emotional intelligence in the structured employment interview. Tesis Doctoral. Oklahoma State University.

Latham, G. P., Saari, L. M., Pursell, E. D. y Campion, M. A. (1980). The situational interview. Journal of Applied Psychology, 65, 422-427.

Ley Orgánica 3/2007, de 22 de marzo, para La Igualdad Efectiva de Mujeres y Hombres.

Little, J. P., Shonenfelt, E. L. y Brown, R. D. (2000). The situational versus the patterned-behavioural-descriptive interview for predicting customer-service performance. Paper presentado en The $15^{\text {th }}$ Annual Conference of the Society for Industrial and Oranizational Psychology, New Orleans, LA, 14 de Abril.

Mayfield, E. C. (1964). The selection interview: A re-evaluation of published research. Personnel Psychology, 17, 239-260.

*McCarthy, J. M., Van Iddekinge, C. H. y Campion, M. A. (2010). Are highly structured job interviews resistant to demographic similarity effects? Personnel Psychology, $63,325-359$.

McDaniel, M. A., Whetzel, D. L., Schmidt, F. L. y Maurer, S. D. (1994). The validity of employment interviews: A comprehensive review and meta-analysis. Journal of Applied Psychology, 79, 599-616.

Moscoso, S. (2000). Selection interview: A review of validity evidence, adverse impact and applicant reactions. International Journal of Selection and Assessment, 8, 237-247.

Moscoso, S. y Salgado, J. F. (2001). Psychometric properties of a structured behavioral interview to hire private security personnel. Journal of Business and Psychology, 16, 51-59.

*Pintigore, R., Dugoni, B. L., Tindale, R. S. y Spring, B. (1994). Bias against overweight job applicants in a simulated employment interview. Journal of Applied Psychology, 79, 909-917.

Prewett- Livingston, A. J. y Field, H. S. (1999). The employment interview and race: A review. En A. Memon and R. Bull (eds.). Handbook of the Psychology of Interviewing. Chichester: Jonh Wiley and Sons Ltd.

*Pulakos, E. D. y Schmitt, N. (1995). Experience-based and Situational Interview Questions: Studies of validity. Personnel Psychology, 48, 289-308.

*Sacco, J. M., Scheu, C. R., Ryan, A. M. y Schmitt, N. (2003). An investigation of race and sex similarity effects in interviews: A multilevel approach to relational demography. Journal of Applied Psychology, 88, 852-865.

Saez, J. (2007). Diseño y validación de una entrevista conductual estructurada para la selección de agentes de policía local. Revista de Psicología del Trabajo y las Organizaciones, 23, 57-74.

*Saez, J. (2011). Determinantes del desempeño individual en agentes de policía local. Tesis doctoral no publicada. Universidad de Santiago de Compostela. 
Salgado, J. F. (1999). Personnel selection methods. En C.L. Cooper y I.T. Robertson (Eds.). International review of industrial and organizational psychology (Vol. 12, pp. 153). London: John Wiley y Sons.

*Salgado, J. F., Gorriti, M. y Moscoso, S. (2007). La entrevista conductual estructurada y el desempeño laboral en la administración pública española: Propiedades psicométricas y reacciones de justicia. Revista de Psicología del Trabajo y de las Organizaciones, 23, 39-55.

Salgado, J. F. y Moscoso, S. (1995). Validez de las entrevistas conductuales estructuradas. Psicología del Trabajo y las Organizaciones, 11, 9-24.

Salgado, J. F y Moscoso, S. (2002). Comprehensive metaanalysis of the construct validity of the employment interview. European Journal of Work and Organizational Psychology, 11, 299-324.

Salgado, J. F. y Moscoso, S. (2005). La entrevista conductual estructurada de selección de personal: Teoría, práctica y rentabilidad. $2^{\mathrm{a}}$ edición. Madrid: Pirámide.

Salgado, J. F. y Moscoso, S. (2006). Utiliser les entretiens comportementaux structurés pour la sélection du personnel? En C. Lévy-Leboyer, C. Louche y J.P. Rolland (Eds.). RH: Les apports de la psychologie du travail. Vol $l$ (pp 195-207). Paris: Éditions d'Organisation.

Salgado, J. F., Moscoso, S. y Gorriti, M. (2004). Investigaciones sobre la entrevista conductual estructurada (ECE) en la selección de personal en la administración general del País Vasco: Meta-análisis de la fiabilidad. Revista de Psicología del Trabajo y de las Organizaciones, 20, 107139.

Salgado, J. F., Viswesvaran, C. y Ones, D. S. (2001). Predictors used for personnel selection: an overview of construct, methods and technichs. En N. Anderson, D. S.
Ones, H.K. Sinangil y C. Viswesvaran (Eds.), Handbook of Industrial, Work and Organizacional Psychology. Vol 1 (pp 165-199). Londres: Sage.

Schmidt, F. L. y Hunter, J. E. (1998). The validity and utility of selection methods in personnel psychology: Practical and theoretical implications of 85 years of research findings. Psychological Bulletin, 124, 262-274.

Schmidt, F. L. y Rader, M. (1999). Exploring the boundary conditions for interview validity: meta-analytic validity findings for a new interview type. Personnel Psychology, 52, 445-464.

Schmitt, N. (1976). Social and situational determinants of interview decisions: Implications for the employment interview. Personnel Psychology, 29, 79-101.

Schmitt, N., Clause, C. S. y Pulakos, E. D. (1996). Subgroup differences associated with different measures of some common job-relevant constructs. International Review of Industrial and Organizational Psychology, 11, 115-139.

Schüler, H. y Funke, U. (1989). The interview as a multimodal procedure. En E.W. Eder y G. R. Ferris (eds.). The Employment interview: Theory, research and practice. Beverly Hill, CA: Sage.

*Sue-Chan, C. y Latham, G. P. (2004). The situational interview as a predictor of academic and team performance: A study of the mediating effects of cognitive ability and emotional intelligence. International Journal of Selection and Assessment, 12, 312-320.

Ulrich, L. y Trumbo, D. (1965). The selection interview since 1949. Psychological Bulletin, 63, 100-116.

Wagner, R. (1949). The employment interview: A critical review. Personnel Psychology, 2, 17-46.

Wright, O. R. (1969). Summary of research on the selection interview since 1964. Personnel Psychology, 22, 391-413.

Apéndice 1. Tabla Resumen de los datos de los estudios primarios

\begin{tabular}{|c|c|c|c|c|c|c|}
\hline & Entrevista & $N$ & $r$ & $d$ & $r_{x x}$ & $u$ \\
\hline Arvey, Miller, Gould y Burch (1987) & CONV & 558 & -.200 & -.41 & .75 & .72 \\
\hline Berges (2010) & ECE & 226 & -.150 & -.30 & .83 & 1 \\
\hline Goldberg y Cohen (2004) & CONV & 210 & .030 & .06 & .75 & 1 \\
\hline \multirow[t]{4}{*}{ Kluemper (2006) } & SIT & 81 & .120 & .24 & .83 & .91 \\
\hline & ECE & 81 & -.010 & -.02 & .83 & .91 \\
\hline & SIT & 30 & -.320 & -.68 & .83 & .80 \\
\hline & ECE & 30 & -.380 & -.82 & .83 & .80 \\
\hline \multirow[t]{3}{*}{ McCarthy, Van Iddekinge y Campion (2010) } & CONV & 18369 & -.120 & -.24 & .79 & .85 \\
\hline & SIT & 18285 & -.075 & -.15 & .80 & .85 \\
\hline & ECE & 18285 & -.125 & -.25 & .82 & .85 \\
\hline Pintigore, Dugoni, Tindale y Spring (1994) & CONV & 320 & .345 & .69 & .70 & 1 \\
\hline Pulakos y Schmitt (1995) & SIT & 464 & -.025 & -.05 & .83 & 1 \\
\hline Sacco, Scheu, Ryan y Schmitt (2003) & ECE & 12007 & .005 & .01 & .84 & .91 \\
\hline Saez (2011) & ECE & 173 & 0 & 0 & .92 & 1 \\
\hline Salgado, Gorriti y Moscoso (2007) & ECE & 93 & -.095 & -.19 & .02 & .51 \\
\hline Sue-Chan y Latham (2004) & SIT & 75 & -.190 & -.39 & .83 & 1 \\
\hline
\end{tabular}

Nota. ECE=Entrevista Conductual Estructurada; SIT=Entrevista Situacional; CONV=Entrevista Convencional; N=muestra del estudio; r=correlación sexo y entrevista; d=tamaño del efecto diferencias hombres y mujeres; $r_{\mathrm{xx}=}$ fiabilidad de la entrevista; $\mathrm{u}=$ restricción en el rango. 